\title{
Regorafenib Induces Apoptosis and Inhibits Metastatic Potential of Human Bladder Carcinoma Cells
}

\author{
FEI-TING HSU ${ }^{1,2,3,4}$, CHO-CHIN SUN ${ }^{5}$, CHIA-HSING WU ${ }^{6}$, YEN-JU LEE ${ }^{7}$, \\ CHIH-HUNG CHIANG ${ }^{5,6,8^{*}}$ and WEI-SHU WANG ${ }^{9,10,11^{*}}$ \\ ${ }^{1}$ Department of Radiology, School of Medicine, Taipei Medical University, Taipei, Taiwan, R.O.C; \\ ${ }^{2}$ Department of Medical Imaging, ${ }^{3}$ Translational Laboratory, Department of Medical Research, and \\ ${ }^{4}$ Research Center of Translational Medicine, Taipei Medical University Hospital, Taipei, Taiwan, R.O.C; \\ ${ }^{5}$ Department of Urology, Medical Research and Education, \\ Taipei Veterans General Hospital, Yuan-Shan/Su-Ao Branch, Yilan, Taiwan, R.O.C.; \\ ${ }^{6}$ Department of Nursing, Cardinal Tien Junior College of Healthcare and Management, New Taipei City, Taiwan, R.O.C.; \\ ${ }^{7}$ Department of Surgery, Zuoying Branch of Kaohsiung Armed Forces General Hospital, Kaohsiung, Taiwan, R.O.C.; \\ ${ }^{8}$ Department of Urology, National Taiwan University Hospital, Taipei, Taiwan, R.O.C.; \\ ${ }^{9}$ Department of Medicine, and ${ }^{10}$ Cancer Medical Care Center, \\ National Yang-Ming University Hospital, Yilan, Taiwan, R.O.C.; \\ ${ }^{11}$ School of Medicine, National Yang-Ming University Hospital, Taipei, Taiwan, R.O.C.
}

\begin{abstract}
The aim of the present study was to verify the effects of regorafenib on apoptosis and metastatic potential in TSGH 8301 human bladder carcinoma cells in vitro. Cells were treated with different concentration of regorafenib for different periods of time. Effects of regorafenib on cell viability, apoptosis pathways, metastatic potential, and expression of metastatic and anti-apoptotic proteins were evaluated with the 3-(4,5-dimethylthiazol-2-yl)-2,5-diphenyltetrazolium (MTT) assay, flow cytometry, cell migration and invasion assay, and western blotting. We found regorafenib significantly reduced cell viability, cell migration and invasion, and expression of metastatic and anti-apoptotic proteins. In addition, regorafenib significantly induced accumulation of sub-G $G_{1}$ phase cells, loss of mitochondrial membrane potential, and expression of active caspase-3 and caspase-8. These results show that regorafenib not only induces apoptosis, but also inhibits metastatic potential in bladder cancer TSGH 8301 cells in vitro.
\end{abstract}

*These Authors contributed equally to this study.

Correspondence to: Dr. Wei-Shu Wang, Department of Medicine, National Yang-Ming University Hospital, Yilan, Taiwan, R.O.C. Email:11313@ymuh.ym.edu.tw and Dr. Chih-Hung Chiang, Department of Urology, Medical Research and Education, Taipei Veterans General Hospital, Yuan-Shan/Su-Ao Branch, Yilan, Taiwan, R.O.C. E-mail: guchiang@gmail.com

Key Words: Regorafenib, human bladder carcinoma, apoptosis, metastatic potential.
Bladder cancer, the most common malignancy found in the urinary tract, is classified into non-muscle-invasive and muscle-invasive (MIBC) disease (1). The majority of patient mortality is due to metastasis (2). Current treatment strategies for MIBC include radical cystectomy, chemotherapy, and radiotherapy. However, the survival rates of patients with MIBC have not significantly improved in the past decades (3). Therefore, development of new anticancer agents for patients with MIBC is needed.

Several receptor tyrosine kinases are overexpressed in bladder cancer and activate intracellular signaling transduction, resulting in tumor progression. Many tyrosine kinase inhibitors have been shown to reduce cell proliferation and metastasis through blockage of epithelial growth factor receptor (EGFR), vascular endothelial growth factor receptor (VEGFR), and platelet-derived growth factor receptor (PDGFR) signaling pathways in bladder cancer (4). Sorafenib (Nexavar) is a multi-tyrosine kinase inhibitor approved for treatment of advanced renal cell and advanced hepatocellular carcinoma $(5,6)$. Rose et al. found that sorafenib induces apoptosis and inhibits cell migration in bladder cancer cells (6). Regorafenib (Stivarga) is a sorafenib analog approved for treatment of colorectal cancer and gastrointestinal stromal tumors. In a previous study, we found both sorafenib and regorafenib induce apoptosis via suppression of nuclear factor kappa-light-chain-enhancer of activated B cells (NF-kB) activation in hepatocellular carcinoma (HCC) cells $(7,8)$. However, whether regorafenib has the potential to be used in the treatment of bladder 
cancer is unknown. The aim of the present study was to investigate the effect of regorafenib on cell growth and metastatic potential of TSGH-8301 human bladder carcinoma cells in vitro by using MTT assay, flow cytometry, cell migration and invasion assay, and western blotting.

\section{Materials and Methods}

Chemicals and agents. Regorafenib was kindly provided by Bayer Corporation (Whippany, NJ, USA). RPMI-1640 medium, fetal bovine serum (FBS), L-glutamine, and penicillin streptomycin (PS) were purchased from Gibco/Life Technologies (Carlsbad, CA, USA). 3,3'-Dihexyloxacarbocyanine Iodide $\left(\mathrm{DiOC}_{6}\right)$ was bought from Enzo Life Sciences (Farmingdale, NY, USA). RNase was bought from Fermentas (St. Leon-Rot, Baden-Wurttemberg, Germany). Propidium iodide (PI), CaspGLOW ${ }^{\mathrm{TM}}$ Fluorescein Active Caspase-3 Staining Kit, and CaspGLOW ${ }^{\mathrm{TM}}$ Red Active Caspase-8 Staining Kit were obtained from Biovision (Mountain View, CA, USA). MTT was purchased from Sigma-Aldrich (St. Louis, MO, USA). Matrigel and Transwell $(8-\mu \mathrm{m}$ pore size) were purchased from Selleck Chemicals (Houston, TX, USA) and Corning Life Sciences (Tewksbury, MA, USA), respectively. Primary antibody to $\mathrm{X}$-linked inhibitor of apoptosis protein (XIAP) was bought from Thermo Fisher Scientific (Fremont, CA, USA). Primary antibody to $\beta$-actin was purchased from Santa Cruz Biotechnology (Santa Cruz, CA, USA). Primary antibody to induced myeloid leukemia cell differentiation protein (MCL1) was bought from BioVision (Milpitas, CA, USA). Primary antibody to matrix metallopeptidase 9 (MMP9) was obtained from EMD Millipore (Billerica, MA, USA). Cellular FLICE (FADD-like IL1 $\beta$-converting enzyme)-inhibitory protein (C-FLIP) were bought from Cell Signaling Technology, Inc. (Danvers, MA, USA). Secondary antibodies were purchased from Jackson ImmunoResearch (West Grove, PA, USA).

Cell culture. TSGH 8301 human bladder carcinoma cells were used for this study and obtained from Professor Jing-Gung Chung at the Department of Biological Science and Technology, China Medical University, Taichung, Taiwan, ROC. TSGH 8301 cells were incubated in RPMI 1640 medium supplemented with $10 \%$ FBS, 2 mM Lglutamine, $100 \mathrm{U} / \mathrm{ml}$ penicillin, and $100 \mathrm{mg} / \mathrm{ml}$ streptomycin at $37^{\circ} \mathrm{C}$ in a humidified incubator containing $5 \% \mathrm{CO}_{2}(9)$.

3-(4,5-Dimethylthiazol-2-yl)-2,5-diphenyltetrazolium bromide (MTT) assay. TSGH 8301 cells were seeded into 96-well plates at $1 \times 10^{4}$ cells/well, incubated overnight, and then treated with different concentration of regorafenib $(0-50 \mu \mathrm{M}$ in $0.1 \%$ dimethylsulfoxide) for 24 and $48 \mathrm{~h}$. The effect of regorafenib on cell viability of TSGH 8301 cells was evaluated with MTT assay as previously described (5).

Detection of the sub-G $G_{1}$ cell population. TSGH 8301 cells were seeded into 6 -well plates at $5 \times 10^{5}$ cells/well, incubated overnight, and then treated with $30 \mu \mathrm{M}$ regorafenib for 24 and $48 \mathrm{~h}$. Propidium iodide (PI) buffer (40 $\mu \mathrm{g} / \mathrm{ml}$ PI, $100 \mu \mathrm{g} / \mathrm{ml} \mathrm{RNase}$ and $1 \%$ Triton $\mathrm{X}-100$ in PBS) was used to identify cell-cycle distribution. The effect of regorafenib on sub- $\mathrm{G}_{1}$ cell population was analyzed by flow cytometry (FACSCalibur; Becton-Dickinson, Franklin Lakes, NJ, USA) as previously described (8).
Detection of active caspase-3. TSGH 8301 cells were seeded into 6 -well plates at $5 \times 10^{5}$ cells/well incubated overnight, and then treated with $30 \mu \mathrm{M}$ regorafenib for 24 and $48 \mathrm{~h}$. $\operatorname{Asp}\left(\mathrm{OCH}_{3}\right)$ $\mathrm{Glu}\left(\mathrm{OCH}_{3}\right)$-Val-Asp $\left(\mathrm{OCH}_{3}\right)$-fluoromethyl ketone (DEVD-FMK) was conjugated by fluorescein isothiocyanate (FITC) to make a working solution ( $1 \mu \mathrm{l}$ FITC-DEVD-FMK in $300 \mu \mathrm{l}$ PBS), which was used to monitor expression of active caspase-3. The effect of regorafenib on active caspase-3 was analyzed by using flow cytometry (FACSCalibur; Becton-Dickinson) as described by Chiang et al. (10).

Detection of active caspase-8. TSGH 8301 cells were seeded into 6 -well plates at $5 \times 10^{5}$ cells/well, incubated overnight, and then treated with $30 \mu \mathrm{M}$ regorafenib for 24 and $48 \mathrm{~h}$. Z-Ile-Glu(OMe)Thr-Asp(OMe)-FMK (IETD-FMK) was conjugated by sulforhodamine (Red-IETD-FMK) to make a working solution ( $1 \mu \mathrm{l}$ RedIETD-FMK in $300 \mu \mathrm{l}$ PBS), which was used to monitor expression of active caspase- 8 . Effect of regorafenib on active caspase- 8 was analyzed by using flow cytometry (FACSCalibur; BectonDickinson) as described by Chen et al. (11).

Detection of mitochondrial membrane potential $\left(\Psi_{m}\right)$. TSGH 8301 cells were seeded into 6 -well plates at $5 \times 10^{5}$ cells/well, incubated overnight, and then treated with $30 \mu \mathrm{M}$ regorafenib for 24 and $48 \mathrm{~h}$. $\mathrm{DiOC}_{6}$ solution $\left(4 \mu \mathrm{M} \mathrm{DiOC}{ }_{6}\right.$ in $\left.500 \mu \mathrm{l} \mathrm{PBS}\right)$ was used to detect $\Delta \Psi_{\mathrm{m}}$. The effect of regorafenib on $\Delta \Psi_{\mathrm{m}}$ was evaluated by using flow cytometry (FACSCalibur; Becton-Dickinson) as described by Wang et al. (12).

Migration assay. Transwell insert with $8 \mu \mathrm{m}$ pore size was purchased from Corning (Tewksbury, MA, USA). TSGH 8301 cells were seeded into $10 \mathrm{~cm}$ diameter dishes at $3 \times 10^{6}$ cells, incubated overnight, and then treated with different concentration of regorafenib for $48 \mathrm{~h}$. After treatment, $1 \times 10^{6}$ cells were collected by centrifugation and resuspended in $1 \mathrm{ml}$ serum free RPMI-1640 then $100 \mu \mathrm{l}$ cell suspension was put into the apical chamber of the transwell insert, and then incubated for $48 \mathrm{~h}$. One hundred microliters of RPMI-1640 with $10 \%$ serum was added to the basolateral chamber. After 24-h incubation, the migrated cells on the transwell membrane were fixed with a mixture of methanol and acetic acid (3:1) for $15 \mathrm{~min}$ and then stained by $0.5 \%$ crystal violet solution. Migrated cells were photographed under a light Nikon ECLIPSE $\mathrm{T} i$-U microscope at $\times 100$ and then quantified using ImageJ software version 1.50 (National Institutes of Health, Bethesda, MD, USA) (13).

Invasion assay. Transwell insert with $8 \mu \mathrm{m}$ pore size was coated with $50 \mu$ l matrigel solution $(25 \mu 1$ matrigel in $25 \mu 1$ serum-free RPMI-1640) and incubated overnight at $37^{\circ} \mathrm{C}$. TSGH 8301 cells were seeded into $10 \mathrm{~cm}$ diameter dishes with $3 \times 10^{6}$ cells, incubated overnight, and then treated with different concentration of regorafenib for $48 \mathrm{~h}$. After treatment, $1 \times 10^{6}$ cells were collected by centrifugation and resuspended in $1 \mathrm{ml}$ serum-free RPMI-1640 then $100 \mu \mathrm{l}$ of cell suspension was put into the apical chamber of the transwell insert and incubated for $48 \mathrm{~h}$. One hundred microliters of RPMI-1640 with $10 \%$ serum was added to the basolateral chamber. After the incubation period, matrigel on the transwell membrane was removed by sterile cotton swab. The invaded cells on the transwell membrane were fixed with mixture of methanol and acetic acid (3:1) for $15 \mathrm{~min}$ and then stained by 


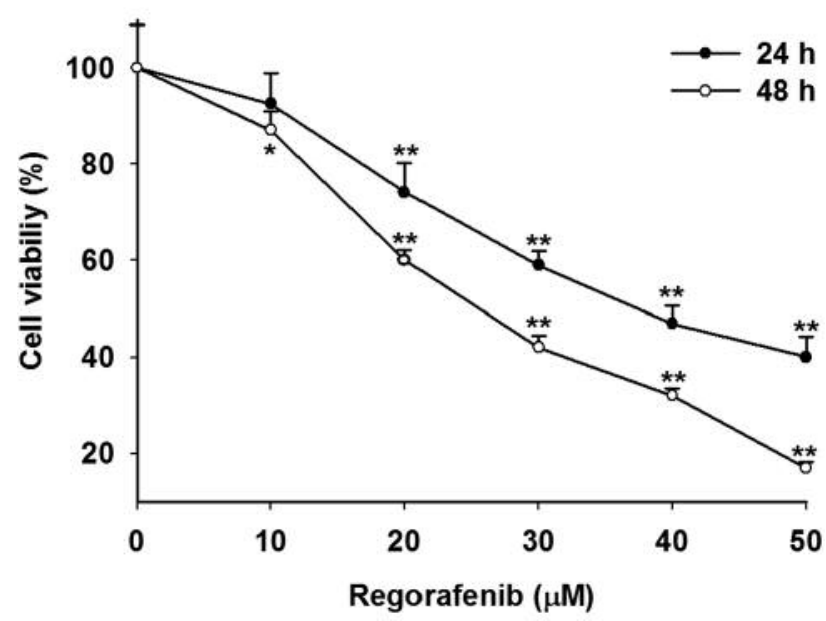

Figure 1. Effect of regorafenib on cell viability of bladder cancer TSGH 8301 cells. Cells were treated with different concentration of regorafenib for 24 and $48 \mathrm{~h}$. Cell viability was evaluated with MTT assay. Significantly different at $* p<0.05$ and $* * p<0.01$ as compared to the control group. Results are presented as the mean \pm standard error.

$0.5 \%$ crystal violet solution. The invaded cells were photographed under a light Nikon ECLIPSE T $i$-U microscope at $\times 100$ and then quantified by using ImageJ software version 1.50 (National Institutes of Health) (14).

Western blotting assay. TSGH 8301 cells $\left(3 \times 10^{6}\right)$ were seeded into $10 \mathrm{~cm}$ diameter dishes, incubated overnight, and then treated with $30 \mu \mathrm{M}$ regorafenib for 24 and $48 \mathrm{~h}$. Total cell proteins from each group were extracted by lysis buffer $(50 \mathrm{mM}$ Tris- $\mathrm{HCl} \mathrm{pH} \mathrm{8.0,}$ $120 \mathrm{mM} \mathrm{NaCl}, 0.5 \% \mathrm{NP}-40$, and $1 \mathrm{mM}$ phenylmethanesulfonyl fluoride). The effect of regorafenib on protein expression levels of MMP-9, XIAP, MCL1, and c-FLIP was determined with western blotting assay as described by Chen et al. (15). Protein bands were quantified by using ImageJ software version 1.50 (National Institutes of Health).

Statistical analysis. Student's $t$-test was used to test significance of difference of means between treatment group and control. Results are presented as mean \pm standard error. Statistical significance was achieved if $p<0.05$. Three independent repeats of each experiment were performed.

\section{Results}

Regorafenib induces cytotoxicity in TSGH 8301 cells. We used the MTT assay to evaluate the effect of regorafenib on cell viability in TSGH 8301 cells. We found that regorafenib significantly inhibited tumor cell growth in dose- and timedependent manners. Regorafenib (10-50 $\mu \mathrm{M})$ significantly reduced cell viability by $5-60 \%$ and $10-80 \%$ at 24 and $48 \mathrm{~h}$ after treatment, respectively, compared to the control (Figure 1).
Regorafenib triggers intrinsic and extrinsic apoptotic pathways in TSGH 8301 cells. We used flow cytometric apoptosis assay to investigate regorafenib-induced apoptotic pathways. We found the regorafenib significantly induced accumulation of cells in the sub- $\mathrm{G}_{1}$ phase and expression of active caspase-3 in TSGH 8301 cells (Figure 2A and B). Regorafenib also significantly elicited expression of active caspase- 8 and loss of $\Psi_{m}$ by $26-53 \%$ and $28-50 \%$, respectively, as compared to the control (Figure $2 \mathrm{C}$ and D).

Regorafenib suppresses cell migration and invasion in TSGH 8301 cells. Cell migration and invasion assays were used to evaluate effect of regorafenib on cell migration and invasion in TSGH 8301 cells. Figure 3A and B indicate regorafenib significantly reduced cell migration and invasion by $95-98 \%$ and $94-98 \%$, respectively, compared to the control.

Regorafenib reduces expression of metastatic and antiapoptotic proteins in TSGH 8301 cells. Western blotting assay was used to verify effect of regorafenib on metastatic and anti-apoptotic proteins expression in TSGH 8301 cells. We found regorafenib significantly reduced protein expression levels of MMP9, XIAP, MCL1, and c-FLIP by $30-90 \%$ compared to the control (Figure 4).

\section{Discussion}

Sorafenib, an anti-HCC agent, has been indicated to induce apoptosis and repress cell migration of bladder cancer cells (6). In a previous study, we found regorafenib, a novel sorafenib derivative, inhibited cell growth and triggered apoptosis in HCC cells in vitro (8). However, whether regorafenib elicits apoptosis and reduces metastatic potential of bladder cancer cells has not been elucidated. Therefore, we investigated the effect of regorafenib on apoptosis and metastatic potential of TSGH 8301 bladder cancer cells.

Many anticancer agents induce extrinsic and intrinsic apoptosis in bladder cancer (16). Expression of active caspase- 8 and loss of $\Psi_{\mathrm{m}}$ are essential for induction of extrinsic and intrinsic apoptotic pathways, respectively (10). Caspase-3, a death protease, is activated in both extrinsic and intrinsic apoptotic pathways. Active caspase-3 modulates cleavage of crucial cellular proteins and formation of apoptotic DNA fragmentation (17). Karamitopoulou et al. suggested active caspase- 3 expression in patients with bladder cancer to be a positive prognostic factor with expected 1-year longer survival than those without expression (18). We found that regorafenib triggered the expression of both active caspase- 3 and caspase- 8 together with loss of $\Psi_{\mathrm{m}}$ in TSGH 8301 cells (Figure 2). Anti-apoptotic proteins including XIAP, MCL-1, and c-FLIP are disruptors of anticancer agent-induced apoptosis (8). High protein levels of XIAP and c-FLIP are also linked to 
A
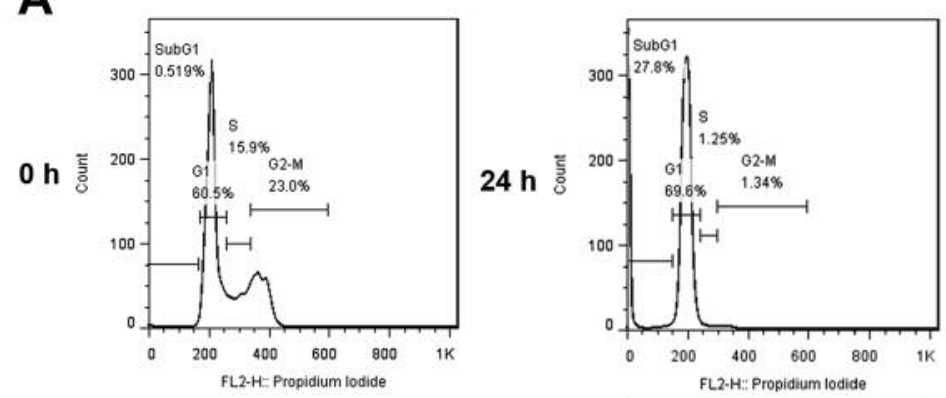

$48 \mathrm{~h}$
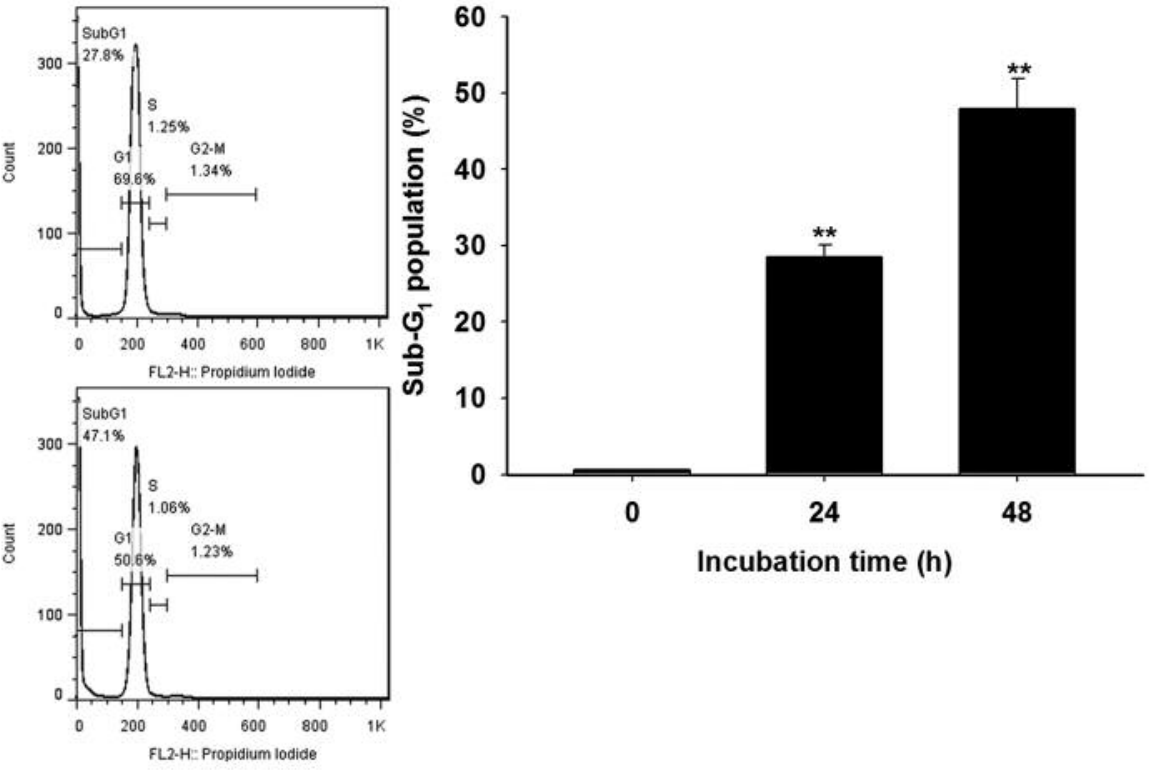

B
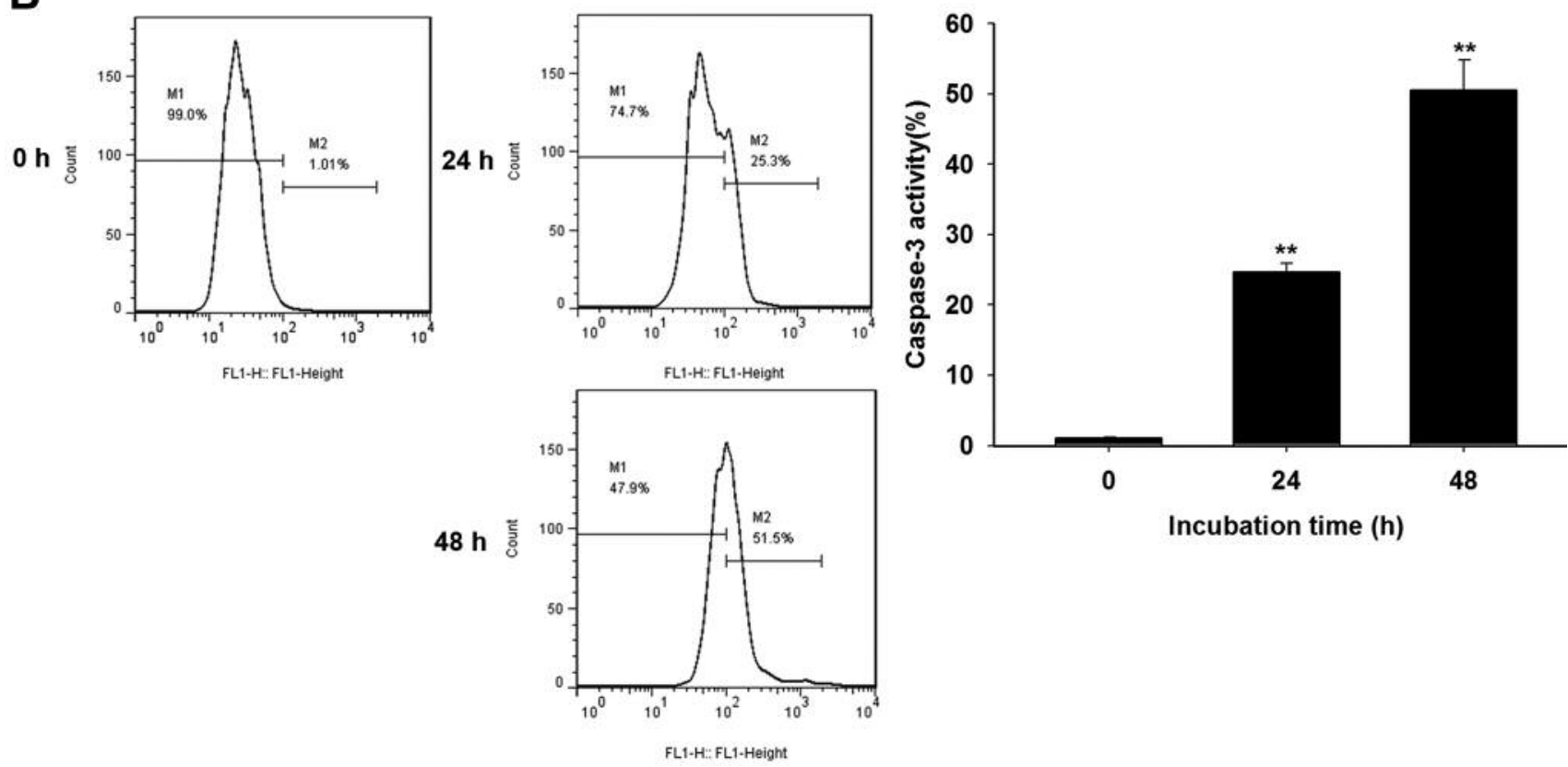

poor prognosis in patients with bladder cancer (19-20). Our data indicate that regorafenib inhibited expression of XIAP, MCL-1, and c-FLIP in in TSGH 8301 cells (Figure 4).

Mortality of patients with MIBC, which comprises 10$20 \%$ of all patients with bladder cancer at diagnosis, is significantly attributed to tumor metastasis, accounting for
$50 \%$ of patients who die from bladder cancer (21). Degradation of extracellular matrix by MMPs is associated with tumor invasion and metastasis. MMP9, $92 \mathrm{kDa}$ gelatinase $\mathrm{B}$, is overexpressed in invasive bladder cancer and related to poor prognosis (22). Our data show that regorafenib reduced the expression of MMP9 (Figure 4) and 

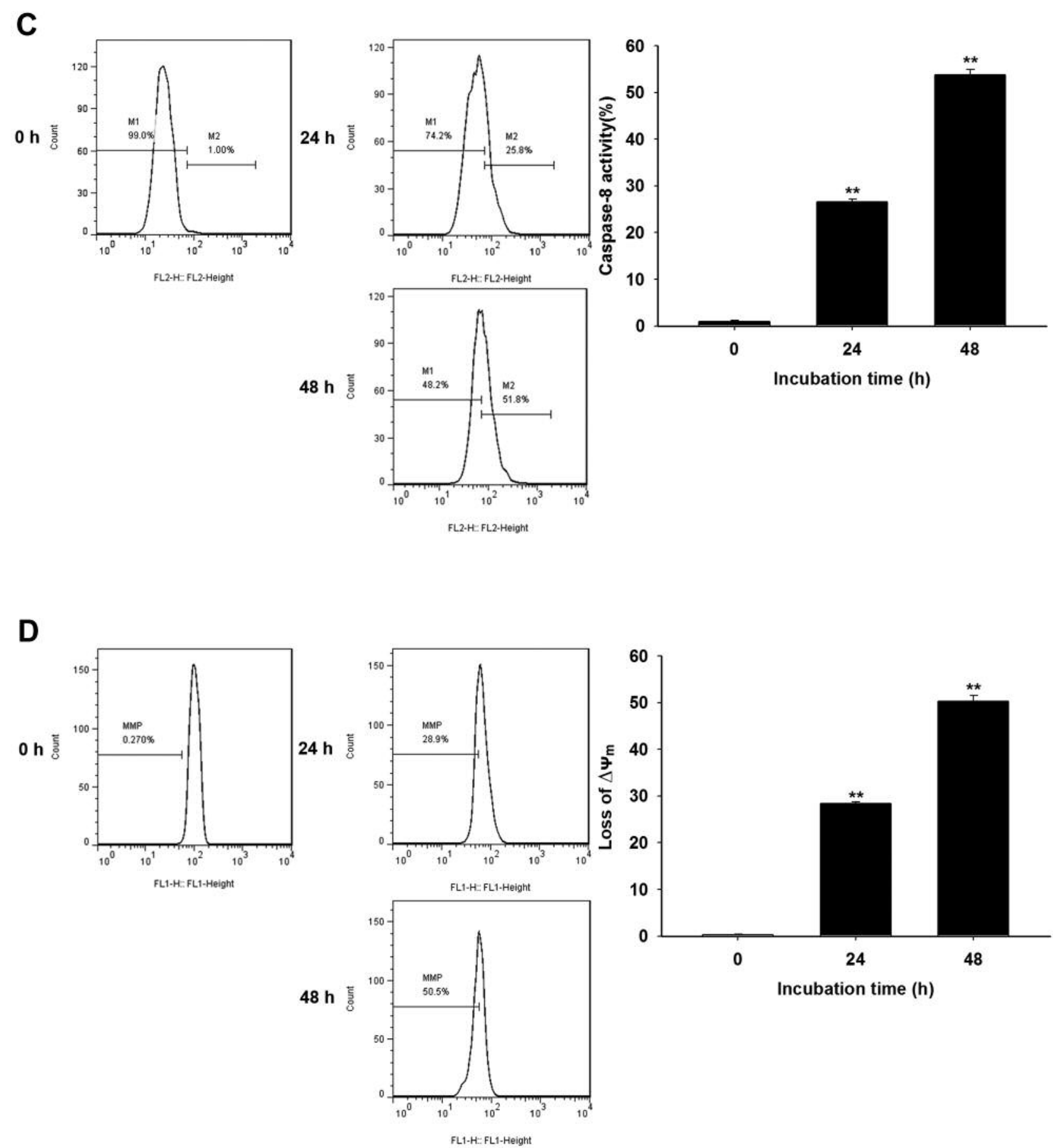

Figure 2. Effect of regorafenib on apoptosis pathways in TSGH 8301 cells. Cells were treated with $30 \mu \mathrm{M}$ regorafenib for 24 and 48 h. Change of sub- $G_{1}$ population $(A)$, expression of active caspase-3 $(B)$ and caspase- $8(C)$, and mitochondrial membrane potential $\left(\Psi_{m}\right)(D)$ were determined by flow cytometry. ${ }^{*}$ Significantly different at $p<0.01$ compared to the control group. Results are presented as the mean \pm standard error.

cell migration and invasion (Figure 3) in TSGH 8301 cells.

In conclusion, this study revealed that regorafenib not only induced apoptosis, but also inhibited metastatic potential of bladder cancer cells. We suggest that regorafenib may be a potential therapeutic agent for patients with bladder cancer. 

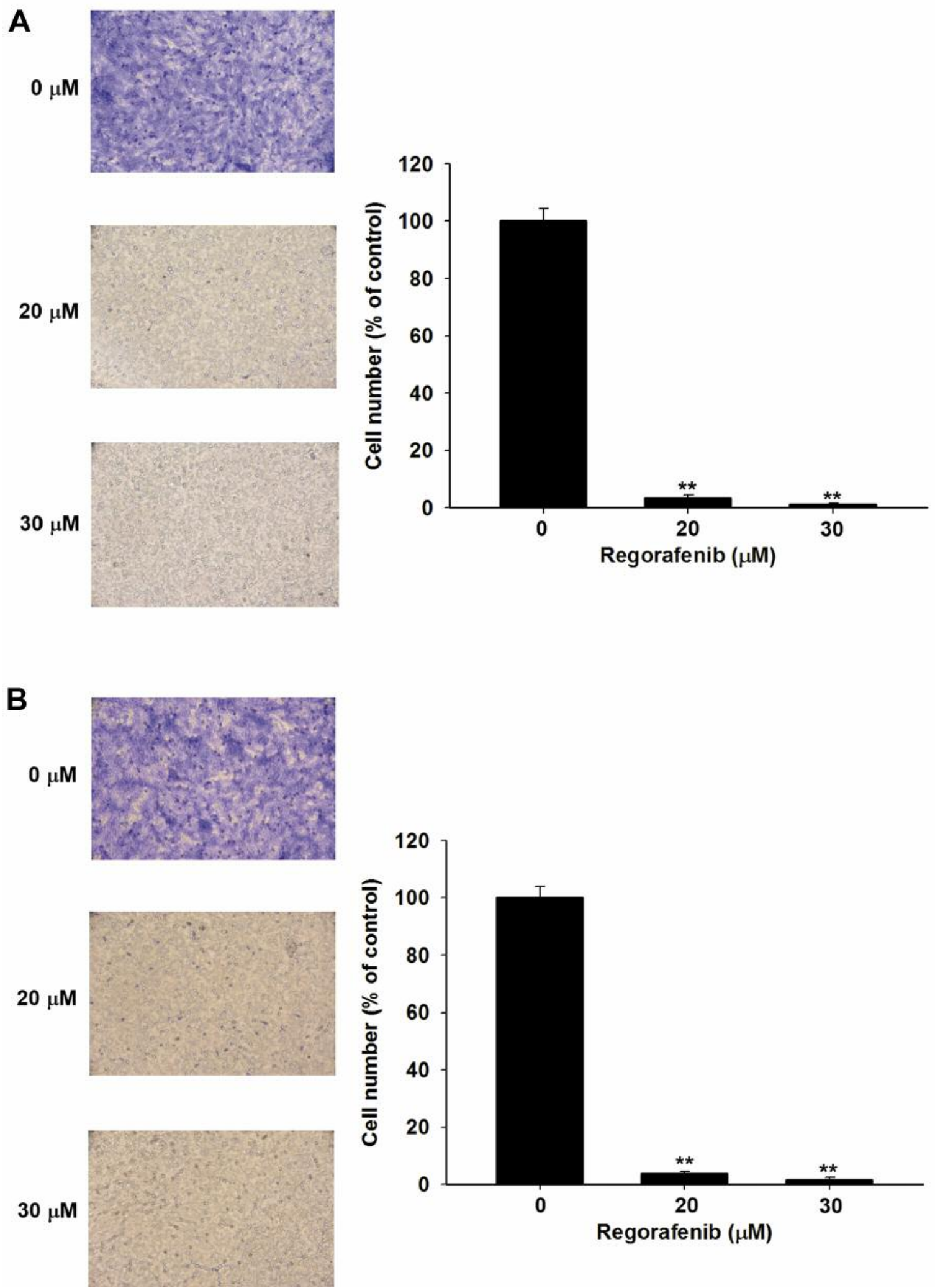

Figure 3. Effect of regorafenib on cell migration and invasion of TSGH 8301 cells. Cells were treated with different concentration of regorafenib for 48 h. Procedures after treatment were in detail described in materials and methods. (A) Cell migration. (B) Cell invasion. **Significantly different at $p<0.01$ compared to the control group. Results are presented as the mean \pm standard error. 


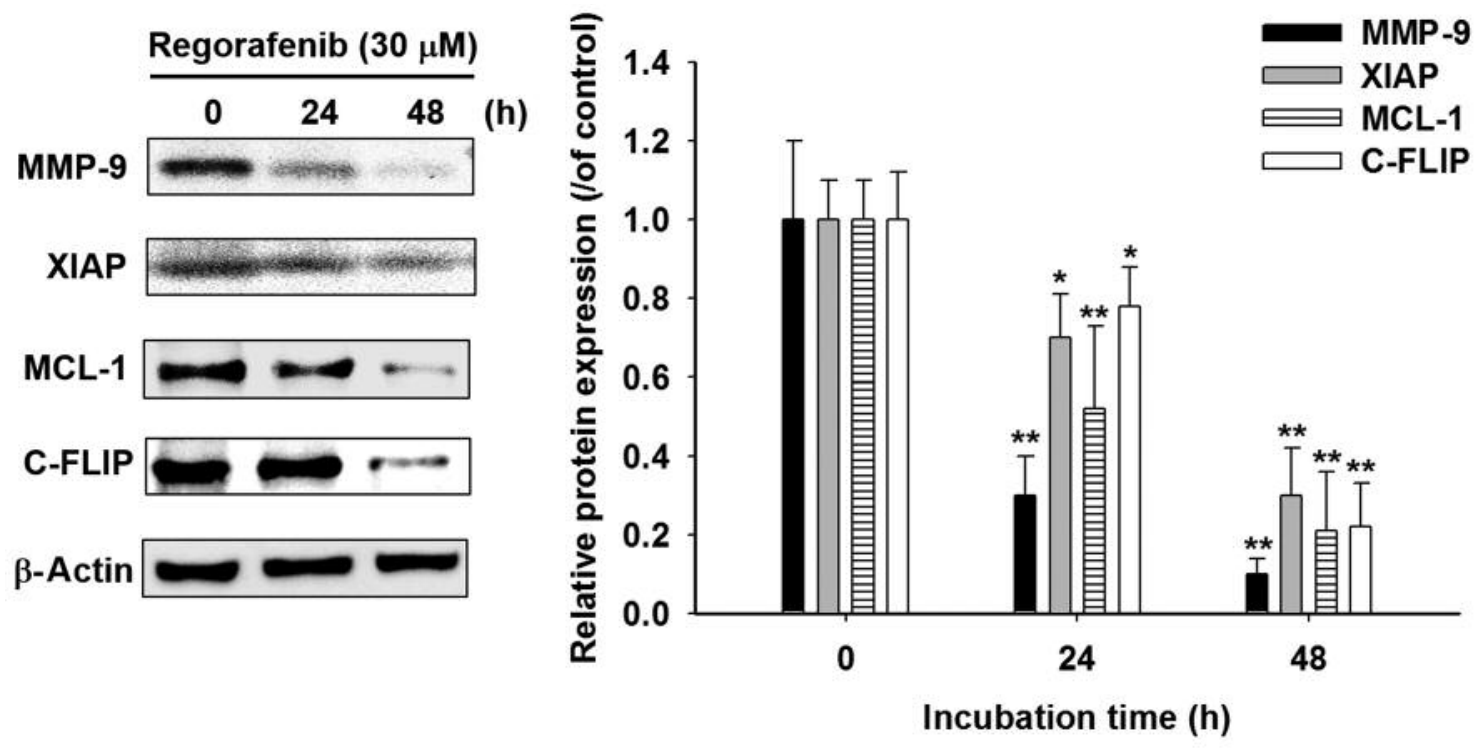

Figure 4. Effect of regorafenib on protein levels of matrix metallopeptidase 9 (MMP9), X-linked inhibitor of apoptosis protein (XIAP), induced myeloid leukemia cell differentiation protein (MCL-1), and cellular FLICE (FADD-like IL1ß-converting enzyme)-inhibitory protein (c-FLIP) in TSGH 8301 cells. Cells were treated with $30 \mu \mathrm{M}$ regorafenib for 24 and $48 \mathrm{~h}$. Change in protein levels was evaluated using western blotting. Significantly different at $* p<0.05$ and $* * p<0.01$ compared to the control group. Results are presented as the mean \pm standard error.

\section{Acknowledgements}

The study was supported by Taipei Medical University/Taipei Medical University Hospital (grant no. 104TMU-TMUH-23, 105TMU-TMUH-23 and TMU105-AE1-B49) and Taipei Veterans General Hospital, Yuan-Shan branch (grant no. YSVH10601). The Authors acknowledge the technical services provided by Clinical Medicine Research Laboratory of National Yang-Ming University Hospital. The Authors also thank for the Translational Laboratory, Department of Medical Research, Taipei Medical University Hospital for their support.

\section{References}

1 Park JC, Citrin DE, Agarwal PK and Apolo AB: Multimodal management of muscle-invasive bladder cancer. Curr Probl Cancer 38: 80-108, 2014.

2 Inoue K, Slaton JW, Karashima T, Yoshikawa C, Shuin T, Sweeney P, Millikan R and Dinney CP: The prognostic value of angiogenesis factor expression for predicting recurrence and metastasis of bladder cancer after neoadjuvant chemotherapy and radical cystectomy. Clin Cancer Res 6: 4866-4873, 2000.

3 Azevedo R, Ferreira JA, Peixoto A, Neves M, Sousa N, Lima A and Santos LL: Emerging antibody-based therapeutic strategies for bladder cancer: A systematic review. J Control Release 214: 40-61, 2015.

4 Wallerand H, Robert G, Bernhard JC, Ravaud A and Patard JJ: Tyrosine-kinase inhibitors in the treatment of muscle-invasive bladder cancer and hormone refractory prostate cancer. Arch Esp Urol 63: 773-787, 2010.
5 Chiang IT, Liu YC, Wang WH, Hsu FT, Chen HW, Lin WJ, Chang WY and Hwang JJ: Sorafenib inhibits TPA-induced MMP9 and VEGF expression via suppression of ERK/NF-kB pathway in hepatocellular carcinoma cells. In Vivo 26: 671-681, 2012.

6 Rose A, Grandoch M, vom Dorp F, Rübben H, Rosenkranz A, Fischer JW and Weber AA: Stimulatory effects of the multikinase inhibitor sorafenib on human bladder cancer cells. Br J Pharmacol 160: 1690-1698, 2010.

7 Hsu FT, Liu YC, Chiang IT, Liu RS, Wang HE, Lin WJ and Hwang JJ: Sorafenib increases efficacy of vorinostat against human hepatocellular carcinoma through transduction inhibition of vorinostat-induced ERK/NF-kB signaling. Int J Oncol 45: 177-188, 2014.

8 Tsai JJ, Pan PJ and Hsu FT: Regorafenib induces extrinsic and intrinsic apoptosis through inhibition of ERK/NF-kB activation in hepatocellular carcinoma cells. Oncol Rep 37: 1036-1044, 2017.

9 Huang YP, Ni CH, Lu CC, Chiang JH, Yang JS, Ko YC, Lin JP, Kuo JH, Chang SJ and Chung JG: Suppressions of Migration and Invasion by Cantharidin in TSGH-8301 Human bladder carcinoma cells through the inhibitions of matrix metalloproteinase-2/-9 signaling. Evid Based Complement Alternat Med 2013: 190281, 2013.

10 Chiang IT, Chen WT, Tseng CW, Chen YC, Kuo YC, Chen BJ, Weng MC, Lin HJ and Wang WS: Hyperforin inhibits cell growth by inducing intrinsic and extrinsic apoptotic pathways in hepatocellular carcinoma cells. Anticancer Res 37: 161-167, 2017.

11 Chen TC, Lai KC, Yang JS, Liao CL, Hsia TC, Chen GW, Lin JJ, Lin HJ, Chiu TH, Tang YJ and Chung JG: Involvement of reactive oxygen species and caspase-dependent pathway in berberine-induced cell-cycle arrest and apoptosis in C6 rat glioma cells. Int J Oncol 34: 1681-1690, 2009. 
12 Wang WH, Chiang IT, Ding K, Chung JG, Lin WJ, Lin SS and Hwang JJ: Curcumin-induced apoptosis in human hepatocellular carcinoma j5 cells: critical role of ca $(+2)$-dependent pathway. Evid Based Complement Alternat Med 2012: 512907, 2012.

$13 \mathrm{Wu}$ ZY, Lien JC, Huang YP, Liao CL, Lin JJ, Fan MJ, Ko YC, Hsiao YP, Lu HF and Chung JG: Casticin Inhibits A375.S2 Human melanoma cell migration/invasion through downregulating NF-kB and matrix metalloproteinase-2 and -1. Molecules 21: 384, 2016.

14 Chen JH, Chen WL and Liu YC: Amentoflavone induces antiangiogenic and anti-metastatic effects through suppression of NF-kB activation in MCF-7 cells. Anticancer Res 35: 66856693, 2015.

15 Chen JC, Chuang HY, Hsu FT, Chen YC, Chien YC and Hwang JJ: Sorafenib pretreatment enhances radiotherapy through targeting MEK/ERK/NF-kB pathway in human hepatocellular carcinoma-bearing mouse model. Oncotarget 7: 85450-85463, 2016.

16 Konac E, Varol N, Kiliccioglu I and Bilen CY: Synergistic effects of cisplatin and proteasome inhibitor bortezomib on human bladder cancer cells. Oncol Lett 10: 560-564, 2015.

17 Porter AG and Jänicke RU: Emerging roles of caspase-3 in apoptosis. Cell Death Differ 6: 99-104, 1999.

18 Karamitopoulou E, Rentsch CA, Markwalder R, Vallan C, Thalmann GN and Brunner T: Prognostic significance of apoptotic cell death in bladder cancer: a tissue microarray study on 179 urothelial carcinomas from cystectomy specimens. Pathology 42: 37-42, 2010.
$19 \mathrm{Li} \mathrm{M}$, Song T, Yin ZF and Na YQ: XIAP as a prognostic marker of early recurrence of nonmuscular invasive bladder cancer. Chin Med J (Engl) 120: 469-473, 2007.

20 Korkolopoulou P, Goudopoulou A, Voutsinas G, Thomas-Tsagli E, Kapralos P, Patsouris E and Saetta AA: c-FLIP expression in bladder urothelial carcinomas: its role in resistance to FASmediated apoptosis and clinicopathologic correlations. Urology 63: 1198-1204, 2004.

21 Reis ST, Leite KR, Piovesan LF, Pontes-Junior J, Viana NI, Abe DK, Crippa A, Moura CM, Adonias SP, Srougi M and Dall'Oglio MF: Increased expression of MMP-9 and IL-8 are correlated with poor prognosis of bladder cancer. BMC Urol 12: $18,2012$.

22 Vasala K, Pääkko $\mathrm{P}$ and Turpeenniemi-Hujanen T: Matrix metalloproteinase-9 (MMP-9) immunoreactive protein in urinary bladder cancer: a marker of favorable prognosis. Anticancer Res 28: 1757-1761, 2008. 